\title{
Characteristics of human papillomaviruses distribution in Guizhou Province, China
}

\author{
Zuyi Chen ${ }^{1,4+}$, Qiongyao $\mathrm{Li}^{2 \dagger}$, Qiong Huang ${ }^{3}$, Huaqing Liu ${ }^{3}$, Hongwu Jiang ${ }^{1,2}$, Zehui Chen ${ }^{1 *}$, Zhengyuan An ${ }^{1}$ and \\ Qingfang Luo,
}

\begin{abstract}
Background: Human papillomavirus (HPV) is one of the most common sexually transmitted viruses. Data about HPV infection in Guizhou is limited.

Methods: 56,768 cervical samples were collected and genotyped for 15 main high risk and 6 main low risk HPV types.

Results: 16.95\% (9623/56768) of samples were HPV positive; 90.70\% (8728/9623) of HPV positive women were infected by high risk HPV. High risk and high risk mix infection (1458; 70.85\%) was the most common mix HPV infection type. The highest HPV detection rate was found in age group 41-45 years old (detection rate $=17.89 \%$ ) $(X 2=204.77 ; P<0.001)$; the highest within-group HPV infection rates were found in the $\leq 20(25.62 \%)$ and $\geq 61$ (24.67\%) years old age groups, the lowest within-group HPV infection rate was found in the 31-35 years old age group (15.02\%). The highest mix infection proportions were found in the $\geq 61(36.06 \%)$ and $\leq 20(33.63 \%)$ years old age groups $(x 2=111.21 ; P<0.001)$, the lowest mix infection proportion was found in the $41-45(17.42 \%)$ years old age group. The highest high risk infection proportions were found in the 26-30 (92.98\%), $\geq 61$ (92.68\%), and 36-40 $(92.16 \%)$ years old age groups $(x 2=31.72 ; P<0.001)$, the lowest high risk infection proportion was found in the $\leq 20$ (84.96\%) years old age group. HPV infection rates varied with seasons in Guizhou.
\end{abstract}

Conclusions: Characteristics of HPV distribution in Guizhou were identified. There were significant differences in HPV distribution among age groups, prevention strategies should be adjusted according to the characteristics.

Keywords: Human papillomavirus, Season, Age group, Distribution

\section{Introduction}

Human papillomavirus (HPV) is associated with varied epithelial lesions, including cervical intraepithelial neoplasia and genital warts [1]. Cervical cancer is one of the most common malignant tumors among women worldwide, genital warts is also very prevalent among women; each year about 500,000 new cases of cervical cancer and 1 million new cases of genital warts were diagnosed worldwide [2, 3].

More than $200 \mathrm{HPV}$ types have been identified until now (pave.niaid.nih.gov). "High-risk" and "low-risk" were used to distinguish different HPV types according to their risks in causing malignant tumors. HPV 16, 18, 31,

* Correspondence: czhtyb@163.com

${ }^{\dagger}$ Zuyi Chen and Qiongyao Li contributed equally to this work.

'Department of Laboratory Medicine, Affiliated Hospital of Zunyi Medical

University, Zunyi, Guizhou, People's Republic of China

Full list of author information is available at the end of the article
$33,35,39,45,51,52,53,56,58,59,66,68,73$, and 83 are common high risk types; HPV 6, 11, 40, 42, 43, 44, $54,61,70,72$, and 81 are common low risk types [4].

HPV distribution varies geographically considerably. Effective HPV prevention and treatment strategies should be specialized based on local HPV distribution characteristics [5]. There is little data about HPV distribution characteristics in Guizhou, China. Guizhou is a province with 36 million people (data from National Bureau of Statistics of China, http://www.stats.gov.cn/). This study aimed to investigate HPV distribution characteristics in Guizhou, China, to afford data for HPV prevention and treatment locally.

\section{Methods and materials}

From January 1, 2016 to December 31, 2017, cervical swabs were obtained from patients at the Affiliated 
Hospital of Zunyi Medical University. Women over 18 years old with visible cervical lesions and/or HPV-related diseases (e.g. cervical intraepithelial neoplasia) were eligible for inclusion. The present study was approved by the Affiliated Hospital of Zunyi Medical University Ethics Committee, the approval number was ZYFYLS2018(81). Before sample collection, informed consent was obtained.

Cervical specimens were collected by cervical brush and stored in preservative buffer solution $(\mathrm{NaCl} 9 \mathrm{~g}$, sodium benzoate $10 \mathrm{~g}, \mathrm{H}_{2} \mathrm{O} 1 \mathrm{~L}$ ) at $-20^{\circ} \mathrm{C}$. Cervical specimens were detected for HPV Genotyping by a Kit (Human Papillomavirus Genotyping Kit; Yaneng Bioscience, Shenzhen, China) according to the instruction. Positive and negative controls were included in all experiments.

SPSS version 19 (IBM, Armonk, NY, USA) was used for data analysis. The Pearson $\chi^{2}$ test was used to confirm the results. $P<0.05$ was considered statistically significant.

\section{Results}

In total, 56,768 samples (mean age $39.45 \pm 9.88$ years old) were collected and detected for HPV genotyping, $16.95 \%$ (9623/56768) of samples were HPV positive; 90.70\% (8728/9623) of HPV positive patients were infected by high risk HPV. HPV detection number was 12, 378, 87.66\% (10,850/12378) were high-risk HPV types. Detection number and detection rate of high-risk types were HPV52 (2206; 17.82\%), HPV16 (1713; 13.84\%), HPV58 (1435; 11.59\%), HPV53 (986; 7.97\%), HPV39 (923; 7.46\%), HPV51 (723; 5.84\%), HPV18 (534; 4.31\%), HPV33 (523; 4.23\%), HPV68 (456; 3.68\%), HPV31 (380; $3.07 \%)$, HPV66 (309; 2.50\%), HPV56 (238; 1.92\%), HPV59 (210; 1.70\%), HPV45 (110; 0.89\%), and HPV35 (104; $0.84 \%)$. Detection number and detection rate of low-risk types were HPV81 (769; 6.21\%), HPV6 (262;
2.12\%), HPV11 (241; 1.95\%), HPV44 (145; 1.17\%), HPV43 (63; 0.51\%), and HPV42 (48; 0.39\%).

Women were divided into different age groups. The highest HPV detection rate was found in age group $41-45$ years old (detection rate $=17.89 \%)\left(\chi^{2}=204.77\right.$; $P<0.001$ ) (see Table 1 ); but the highest within-group HPV infection rates were found in the $\leq 20(25.62 \%)$ and $\geq 61(24.67 \%)$ years old age groups, the lowest within-group HPV infection rate was found in the 3135 years old age group (15.02\%) (see Table 1). The highest mix infection proportions were found in the $\geq 61(36.06 \%)$ and $\leq 20(33.63 \%)$ years old groups $(\chi 2=$ 111.21; $P<0.001)$, the lowest mix infection proportion was found in the 41-45 (17.42\%) years old age group (see Table 2). The highest high risk infection proportions were found in the $26-30(92.98 \%), \geq 61(92.68 \%)$, and 36-40 (92.16\%) years old groups $\left(\chi^{2}=31.72 ; P<\right.$ $0.001)$, the lowest high risk infection proportion was found in the $\leq 20(84.96 \%)$ years old age group (see Table 2).

The number and detection rate of different HPV types detected per patient were listed in Table 3. At most eight infection was found. High risk and high risk mix infection (1458; 70.85\%) was the most common mix HPV infection type with overriding advantage (see Table 3).

HPV positive rates of different seasons were analyzed. Months were divided into Spring (March, April and May), Summer (June, July and August), Autumn (September, October and November) and Winter (January, February and December) according to the standard QX/T1522012 of China Meteorological Information Center (data.cma.cn). We found that positive rate of HPV varied with seasons. The highest and lowest $\left(x^{2}=\right.$ 39.46.; $P<0.001)$ HPV positive rates were found in Spring (2699/14600; 18.49\%) and Autumn (2148/ 13601; 15.79\%), respectively (see Table 4).

Table 1 Detection rate and positive rate of patients in each age group

\begin{tabular}{|c|c|c|c|c|c|}
\hline age & Positive number & Negative number & Total & Detection rate & Positive rate \\
\hline$\leq 20$ & 113 & 328 & 441 & $1.17 \%$ & $25.62 \%$ \\
\hline $21-25$ & 739 & 2807 & 3546 & $7.68 \%$ & $20.84 \%$ \\
\hline $26-30$ & 1410 & 6765 & 8175 & $14.65 \%$ & $17.25 \%$ \\
\hline $31-35$ & 1285 & 7273 & 8558 & $13.35 \%$ & $15.02 \%$ \\
\hline $36-40$ & 1530 & 8280 & 9810 & $15.90 \%$ & $15.60 \%$ \\
\hline $41-45$ & 1722 & 9331 & 11,053 & $17.89 \%$ & $15.58 \%$ \\
\hline $46-50$ & 1320 & 6469 & 7789 & $13.72 \%$ & $16.95 \%$ \\
\hline $51-55$ & 871 & 3821 & 4692 & $9.05 \%$ & $18.56 \%$ \\
\hline $56-60$ & 278 & 987 & 1265 & $2.89 \%$ & $21.98 \%$ \\
\hline$\geq 61$ & 355 & 1084 & 1439 & $3.69 \%$ & $24.67 \%$ \\
\hline Total & 9623 & 47,145 & 56,768 & $100.00 \%$ & $16.95 \%$ \\
\hline
\end{tabular}

Note: Detection rate means positive number percentage of each age group in total positive number. After rounding of "Detection rate" in each age group, the "Detection rate" arithmetic sum of all age group was not $100.00 \%$ 
Table 2 High risk proportion and mix infection proportion of each age group

\begin{tabular}{lllllll}
\hline age & High risk infection & Only low risk infection & High risk proportion & Mix infection & Single infection & Mix infection proportion \\
\hline$\leq 20$ & 96 & 17 & $84.96 \%$ & 38 & 75 & $33.63 \%$ \\
$21-25$ & 676 & 63 & $91.47 \%$ & 193 & 546 & $26.12 \%$ \\
$26-30$ & 1311 & 99 & $92.98 \%$ & 313 & 1097 & $22.20 \%$ \\
$31-35$ & 1168 & 117 & $90.89 \%$ & 249 & 1036 & $19.38 \%$ \\
$36-40$ & 1410 & 120 & $92.16 \%$ & 272 & 1258 & $17.78 \%$ \\
$41-45$ & 1527 & 195 & $88.68 \%$ & 300 & 1422 & $17.42 \%$ \\
$46-50$ & 1183 & 137 & $89.62 \%$ & 299 & 1021 & $22.65 \%$ \\
$51-55$ & 777 & 94 & $89.21 \%$ & 182 & 689 & $20.90 \%$ \\
$56-60$ & 251 & 27 & $90.29 \%$ & 84 & 194 & $30.22 \%$ \\
$\geq 61$ & 329 & 26 & $92.68 \%$ & 128 & 227 & $36.06 \%$ \\
total & 8728 & 895 & $90.70 \%$ & 2058 & 7565 & $21.39 \%$ \\
\hline
\end{tabular}

Note: High risk infection, Only low risk infection, Mix infection, Single infection mean positive patients number of high risk infection, only low risk infection, mix infection, and single infection, respectively. High risk proportion means high risk infection number percentage of each age group in total number of both high risk infection and only low risk infection. Mix infection proportion means mix infection number percentage of each age group in total number of both mix infection and single infection

\section{Discussion}

This study investigated the HPV distribution characteristics in Guizhou. This is the first HPV distribution study with large sample size in Guizhou. Data presented in this study was useful for HPV detection, prevention and treatment in Guizhou.

HPV16, HPV18, HPV31, HPV58 and HPV52 were the five most common high risk HPV types worldwide; HPV16 and HPV18 account for approximately $70 \%$ of cervical cancer cases [6, 7]. While HPV16, HPV52, HPV18, HPV51 and HPV58 were the five most common

Table 3 Percentage of single infection and mixed infections

\begin{tabular}{llll}
\hline Type & Number & Positive rate & Detection rate \\
\hline single & 7565 & $13.326 \%$ & $78.61 \%$ \\
double & 1561 & $2.750 \%$ & $16.22 \%$ \\
three & 360 & $0.634 \%$ & $3.74 \%$ \\
four & 97 & $0.171 \%$ & $1.01 \%$ \\
five & 25 & $0.044 \%$ & $0.26 \%$ \\
six & 8 & $0.014 \%$ & $0.08 \%$ \\
seven & 6 & $0.011 \%$ & $0.06 \%$ \\
eight & 1 & $0.002 \%$ & $0.01 \%$ \\
Total & 9623 & $16.95 \%$ & $100.00 \%$ \\
HR-HR & 1458 & $2.57 \%$ & $70.85 \%$ \\
HR-LR & 580 & $1.02 \%$ & $28.18 \%$ \\
LR-LR & 20 & $0.04 \%$ & $0.97 \%$ \\
Total & 2058 & $3.63 \%$ & $100.00 \%$ \\
\hline
\end{tabular}

Note: Single, double, three, four, five, six, seven, eight means single, double, three, four, five, six, seven, eight infection, respectively. HR-HR means high-risk and high-risk mixed infection; HR-LR means high-risk and low-risk mixed infection; LR-LR means low-risk and low-risk mixed infection. Positive rate means positive number percentage of each infection type in total patients. Detection rate means positive number percentage of each infection type in total positive number. After rounding, the "Detection rate" arithmetic sum of single, double, three, four, five, six, seven and eight infection was not $100.00 \%$ high risk HPV types in China [6]. In the present study, HPV52, HPV16, HPV58, HPV53 and HPV39 were the five most common high risk HPV types in Guizhou. HPV53, HPV39 and HPV51 were more common than HPV18; HPV53 and HPV51 were rarely reported as the most common types in China recently [8-14]. No HPV vaccine target HPV53, HPV39 and HPV51 until now. So HPV53, HPV39 and HPV51 should be important targets of high risk HPV prevention by detection. HPV vaccine for HPV53, HPV39 and HPV51 should be developed for Guizhou.

HPV6 and HPV11 were the most common low risk HPV types worldwide and in China [9]. But in the present study, we found that HPV81 was the most common low risk type with overwhelming advantage. And what was worse, no HPV vaccine target HPV81 until now. So low risk HPV prevention key target by detection may should be adjusted from HPV6 and HPV11 to HPV81. HPV81 vaccine should be developed for Guizhou.

High-risk HPV persistent infection is the main reason of cervical cancer [15]. In the present study, we found that the high risk HPV infection proportions were up to 90.70 and $92.98 \%$ in all and in $26-30$ years old age group

Table 4 Positive rates of four seasons

\begin{tabular}{llll}
\hline Season & Positive number & Negative number & Positive rate \\
\hline Spring & 2699 & 11,901 & $18.49 \%$ \\
Summer & 2848 & 14,369 & $16.54 \%$ \\
Autumn & 2148 & 11,453 & $15.79 \%$ \\
Winter & 1928 & 9422 & $16.99 \%$ \\
Total & 9623 & 47,145 & $16.95 \%$ \\
\hline
\end{tabular}

Note: Positive number and Negative number mean HPV positive and HPV negative number patients in each season. Positive rate means positive number percentage in total patients of each season 
HPV positive women, respectively. Proportion of high risk HPV infection in Guizhou was much higher than studies in other recent reports in China [8-14].

In the present study, we found that the most prevalent HPV infection age was between 41 and 45 years old (detection rate $=17.89 \%$ ), similar to few recent studies in China $[9,10]$, but different from most studies in and out of China [8, 11-14, 16, 17]. The HPV with-in group positive rate, mix infection proportion and high risk infection proportion were all relatively high in age group $\geq 61$. Ideal prevention strategies for Guizhou population should be developed according to the HPV distribution characteristics of each age group in Guizhou, especially for the age groups with highest HPV with-in group positive rates, mix infection proportions and high risk infection proportions.

Whether HPV mix infections increase the risk of disease is not clear. Antigens vary among different HPV types; cross-defense is not effective enough [18]. HPV mix infection may increase the risk of HPV-related diseases. Compared to similar studies, high risk and high risk HPV mix infection in Guizhou was very high $[8,9]$. Age groups with higher HPV mix infection in the present study may need more attention in HPV prevention in Guizhou.

In this study, we found that there were statistical differences in HPV positive rates among different seasons, indicating that season factor should also be taken in to consideration in HPV prevention in Guizhou.

\section{Conclusion}

HPV52, HPV16, HPV58, HPV53 and HPV39 were the five most common high risk HPV types in Guizhou. HPV81 was the most common low risk type in Guizhou. High risk HPV infection proportion was very high in Guizhou. There were significant differences in HPV distribution among age groups, prevention strategy should be adjusted according to the HPV characteristics of each group. HPV infection rates varied with seasons in Guizhou.

\section{Abbreviation}

HPV: Human papillomavirus

\section{Acknowledgments}

We thank for doctors of obstetrics and gynecology department of Affiliated Hospital of Zunyi Medical University.

\section{Authors' contributions}

ZC, QL and ZC conceived and designed the study. ZC, QL, QH, HL, HJ, ZC, ZA and QL performed the experiments. ZC and QL analyzed the data. ZC and QL wrote the paper. All authors read and approved the final manuscript.

\section{Funding}

This reseach was funded by Affiliated Hospital of Zunyi Medical University (number 201808) and Innovation and Entrepreneurship Training Program for College Students (number ZKDC2018017 and 20195201909).

\section{Availability of data and materials}

All data generated or analyzed during this study are included in this published article.

\section{Ethics approval and consent to participate}

The study was approved by the Ethics Committee of Affiliated Hospital of Zunyi Medical University, the approval number was ZYFYLS2018(81). Before sample collection, a written informed consent was obtained from all the patients or their guardians, and patient/study subject privacy was carefully protected.

\section{Consent for publication}

Not applicable.

\section{Competing interests}

The authors declare that they have no competing interests.

\section{Author details}

'Department of Laboratory Medicine, Affiliated Hospital of Zunyi Medical University, Zunyi, Guizhou, People's Republic of China. ${ }^{2}$ Department of Information Technology, Affiliated Hospital of Zunyi Medical University, Zunyi, Guizhou, People's Republic of China. ${ }^{3}$ Department of Pathology, Affiliated Hospital of Zunyi Medical University, Zunyi, Guizhou, People's Republic of China. ${ }^{4}$ School of Laboratory Medicine, Zunyi Medical University, Zunyi, Guizhou, People's Republic of China.

Received: 14 September 2019 Accepted: 9 October 2019

Published online: 29 October 2019

\section{References}

1. Martins TR, de Oliveira CM, Rosa LR, et al. HPV genotype distribution in Brazilian women with and without cervical lesions: correlation to cytological data[J]. Virol J. 2016;13(1):138

2. Chen Z, Jing Y, Wen Q, et al. E6 and E7 gene polymorphisms in human papillomavirus types-58 and 33 identified in Southwest China[J]. PLoS One. 2017:12(1):e0171140.

3. Scheinfeld N, Lehman DS. An evidence-based review of medical and surgical treatments of genital warts[]]. Dermatology online journal. 2006; 12(3):5-5.

4. Muñoz N, Bosch FX, De Sanjosé S, et al. Epidemiologic classification of human papillomavirus types associated with cervical cancer[]]. N Engl J Med. 2003;348(6):518-27.

5. Chen Z, Jing Y, Wen Q, et al. L1 and L2 gene polymorphisms in HPV-58 and HPV-33: implications for vaccine design and diagnosis[[]. Virol J. 2016;13(1):167.

6. Castellsague X. HPV and cervical Cancer in the world: 2007 report (section I continents and regions)[]]. Vaccine. 2007;25:C1-C26.

7. Ursu RG, Onofriescu M, Nemescu D, et al. HPV prevalence and type distribution in women with or without cervical lesions in the northeast region of Romania[J]. Virol J. 2011:8(1):558.

8. Chen Z, Wang Q, Ding X, et al. Characteristics of HPV prevalence in Sichuan Province, China[J]. Int J Gynecol Obstet. 2015:131(3):277-80

9. Zeng Z, Yang H, Li Z, et al. Prevalence and genotype distribution of HPV infection in China: analysis of 51,345 HPV genotyping results from China's largest CAP certified laboratory[J]. J Cancer. 2016;7(9):1037.

10. Zhao P, Liu S, Zhong Z, et al. Prevalence and genotype distribution of human papillomavirus infection among women in northeastern Guangdong Province of China[J]. BMC Infect Dis. 2018;18(1):204.

11. Zhao R, Zhang WY, Wu MH, et al. Human papillomavirus infection in Beijing, People's Republic of China: a population-based study[]]. Br J Cancer. 2009; 101(9):1635.

12. Chen $X$, Wallin KL, Duan $M$, et al. Prevalence and genotype distribution of cervical human papillomavirus (HPV) among women in urban Tianjin, China[J]. J Med Virol. 2015;87(11):1966-72.

13. Wang $\mathrm{S}$, Wei $\mathrm{H}$, Wang $\mathrm{N}$, et al. The prevalence and role of human papillomavirus genotypes in primary cervical screening in the northeast of China[J]. BMC Cancer. 2012;12(1):160.

14. Chen $Q$, Luo ZY, Lin M, et al. Prevalence and genotype distribution of human papillomavirus infections in women attending hospitals in Chaozhou of Guangdong province[J]. Asian Pac J Cancer Prev. 2012;13(4): 1519-24. 
15. Baboci L, Boscolo-Rizzo P, Holzinger D, et al. Evidence of the causal role of human papillomavirus type 58 in an oropharyngeal carcinoma[J]. Virol J. 2013;10(1):334

16. Anderson LA, O'Rorke MA, Wilson R, et al. HPV prevalence and typedistribution in cervical cancer and premalignant lesions of the cervix: a population-based study from Northern Ireland[J]. J Med Virol. 2016;88(7): 1262-70.

17. Hibbitts $\mathrm{S}$, Rieck GC, Hart K, et al. Human papillomavirus infection: an anonymous prevalence study in South Wales, UK[]]. Br J Cancer. 2006;95(2): 226-32.

18. Karanam B, Jagu S, Huh WK, et al. Developing vaccines against minor capsid antigen L2 to prevent papillomavirus infection[J]. Immunol Cell Biol. 2009; 87(4):287-99.

\section{Publisher's Note}

Springer Nature remains neutral with regard to jurisdictional claims in published maps and institutional affiliations.

Ready to submit your research? Choose BMC and benefit from:

- fast, convenient online submission

- thorough peer review by experienced researchers in your field

- rapid publication on acceptance

- support for research data, including large and complex data types

- gold Open Access which fosters wider collaboration and increased citations

- maximum visibility for your research: over $100 \mathrm{M}$ website views per year

At $\mathrm{BMC}$, research is always in progress.

Learn more biomedcentral.com/submissions 